\title{
Efficient Heuristics for the Dynamic Berth Allocation Problem in Discrete Berthing Locations
}

\author{
Yukiko YAMAKAWA*, Akio IMAI** and Etsuko NISHIMURA**
}

\begin{abstract}
Marine container terminal is a switching facility of containers between sea and land as well as between deepsea and shortsea for sea transportation legs. The overall terminal efficiency highly depends on harmonized operations of complicated and different handling tasks being undertaken at the terminal. One of the most important operational issues is the berth allocation scheduling (or berth allocation problem), because an improper berth schedule forces ships to be berthed far from their containers to be handled in a yard. Such an unproductive service situation is never able to be overcome by any proper operational efforts for other handling tasks. The authors developed heuristics that were implemented using a subgradient optimization technique with a Lagrangian relaxation to the original problem for the dynamic berth allocation problem in discrete locations. This study improves the heuristics by introducing a new procedure to find a better feasible solution in the entire iterative process of subgradient technique.
\end{abstract}

Keywords : logistics, container terminal, berth allocation problem, subgradient optimization

\section{Introduction}

Marine container terminal is a switching facility of containers between sea and land as well as between deepsea and shortsea for sea transportation mode. The overall terminal efficiency highly depends on harmonized operations of complicated and different handling tasks that are undertaken at the terminal. One of the most important operational issues is the berth allocation scheduling (or berth allocation problem), because an improper berth schedule forces ships to be berthed far from their containers to be handled in a yard. Such an unproductive service situation is never able to be overcome by any proper operational efforts for other handling tasks.

The berth scheduling is comprised of two different levels of decision making: tactical and operational ones. The tactical scheduling is the berth template problem that defines berth windows of serving vessels given a long term profile of calling requests from ship operators, while the operation scheduling solves the berth allocation problem (BAP) that assigns a set of calling ships to proper berthing locations with updated arrival and handling cargo volume information. The authors developed heuristics that were implemented using a subgradient optimization technique with a Lagrangian relaxation to the original problem for the dynamic berth allocation problem in discrete locations (DBAPD). This paper proposes an improvement to this subgradient optimization in order to identify a better approximate solution.

The organization of this paper is as follows: the next section summarizes some existing studies on the BAP. Section 3 introduces a mathematical formulation of the DBAPD. The improved solving algorithms are explained in section 4 , which is followed by section 5 that provides numerical experiments. The last section concludes this paper.

\section{Literature review}

The BAP is classified with many schemes. One classification scheme is the start of planning. The static BAP assumes that all calling vessels have already arrived at a port before the start of planning, while the dynamic BAP presumes some of the ships having not yet arrived before the start of planning and going to arrive during the planning horizon.

* Student member Graduate School of Maritime Sciences, Kobe University

** Regular member Graduate School of Maritime Sciences, Kobe University

( T658-0022, 5-1-1 Fukae, Higashinada, Kobe, Japan) imai@maritime.kobe-u.ac.jp 


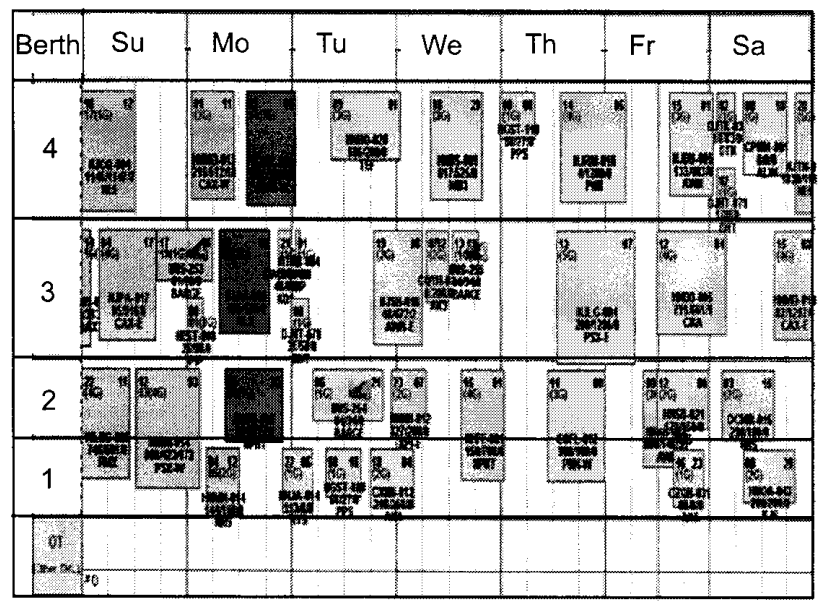

Fig. 1 Berth scheduling example

Another classification scheme is the type of berthing locations. There are two location types: discrete and continuous. In the former for the dynamic BAP (DBAPD), only one ship can be served at a berth at a time, while in the latter (DBAPC) ships are berthed at any quay locations ignoring the berth border. So, the DBAPC leads to a more efficient quay usage.

The DBAPD takes a short computation time but its solution may not be efficient when directly applied to a terminal because of unused quay space. The DBAPC is more flexible and generates a much more productivity than the DBAPD, because the entire quay can be fully utilized without berth boundaries for vessel berthing. The DBAPC is, therefore, more effective than the DBAPD especially when the terminal is congested with a lot of calling vessels. However, the DBAPC is more complicated than the DBAPD and needs a long computation time.

The DBAPD was first addressed by pioneering papers of Imai et al. ${ }^{(10),(11)}$, which are followed by other papers ${ }^{(1),(2),(4),(5)-(7),(9),,(12)-(13),(15)-(16),(22),(24),(26),(28)}$. There are also a lot of papers ${ }^{(3)-(8),(14),(17)-(21),(23),(25),(27)}$, which deal with the DBAPC. Most of them solve the DBAPC as a cutting stock problem because of similar characteristics.

As mentioned before, the DBAPC assures a more berth productivity than the DBAPD. But, the latter can be practically useful since major terminals serve a number of large calling vessels. For a reference purpose, Fig. 1 shows a berth schedule at Hanjin Container Terminal of the Busan New Port. The horizontal axis shows the chronological time (days) while the vertical axis shows berths. There are four berths, while berths 1 and 2 can be used together to provide the same size as berths 3 or 4 . The boxes in the figure indicate calling ship's berth windows, each of which has calling information such as ETA (estimated time of arrival), ETD (estimated time of departure) and ship name. As illustrated, the entire quay is divided into three berths (assuming the entire space of berth 1 and 2 has the same size as berths 3 or 4). Except for few small feeder vessels, all ships occupy a whole berth and the idle quay space is not fully used. For this reason we deal with DBAPD.

\section{Formulations}

\subsection{DBAPD formulation}

The DBAPD is first proposed in Imai et al. ${ }^{(1)}$. The DBAPD formulation in this paper is a slightly modified version from the one in Imai et al. ${ }^{(11),(13)}$. The original formulation assumes that the ship service order is numbered in ascending order from the first ship to be served. In this paper, the reverse order is adopted, i.e., the ship served last is the one to be served as the first ship (service order one) in the model, while the first ship is the one served as the $N$ th ship (service order $N$ ) where $N$ ships are calling at the terminal. This service order scheme results in a simpler formulation than the original one that was proposed in Imai et al. ${ }^{(11),(13)}$. Note, however, that this simplification does not affect the solution quality, since these two types of formulation in different forms turn to be the same model structure.

The formulation is as follows:

[DBAPD] Minimize $\sum_{i \in B} \sum_{j \in V} \sum_{k \in U}\left\{k C_{i j}+S_{i}-A_{j}\right\} x_{i j k}$

subject to

$$
+\sum_{i \in B} \sum_{j \in W_{i}} \sum_{k \in U} k y_{i j k}
$$

$$
\begin{aligned}
& \sum_{j \in V} x_{i j k} \leq 1 \quad \forall j \in V \\
& \sum_{i \in B}^{j \in V} \sum_{k \in U} x_{i j k}=1 \quad \forall i \in B, k \in U \\
& \sum_{l \in V} \sum_{m \in P_{k}}\left(C_{i l} x_{i l m}+y_{i l m}\right)+y_{i j k}-\left(A_{j}-S_{i}\right) x_{i j k} \geq 0 \\
& \forall i \in B, j \in W_{i}, k \in U \\
& x_{i j k} \in\{0,1\} \quad \forall i \in B, j \in V, k \in U \\
& y_{i j k} \geq 0 \quad \forall i \in B, j \in V, k \in U \\
& i(=1, \ldots, I) \in B \quad: \text { set of berths } \\
& j(=1, \ldots, T) \in V \quad \text { : set of ships } \\
& k(=1, \ldots, T) \in U: \text { set of service orders } \\
& A_{j}: \text { arrival time of } \operatorname{ship} j
\end{aligned}
$$


$P_{k}$ : subset of $U$ such that $P_{k}=\{p \mid p>k \in U\}$

$S_{i}$ : time when berth $i$ becomes idle for the planning horizon

$W_{i}$ : subset of ships for berth $i$ with $A_{j} \geq S_{i}$

$C_{i j}$ : handling time spent by ship $j$ at berth $i$

$x_{i j k}:=1$ if ship $j$ is served as the $k$ th ship from the last at berth $i$, and $=0$ otherwise

$y_{i j k}$ : idle time of berth $i$ between the departure of the $(k+1)$ th ship from the last and the arrival of the $k$ th ship from the last when ship $j$ served as the $k$ th ship from the last

The decision variables are $x_{i j k} \mathrm{~s}$ and $y_{i j k} \mathrm{~s}$. Objective (1) minimizes the total service time. Constraint set (2) ensures that every ship must be served at some berth in any order of service. Constraints (3) enforce that every berth serves up to one ship at any time. Constraints (4) assure that ships are served after their arrival. Notice that the entire number of service orders is more than the number of vessels, $T$. For the detailed derivation of objective (1) and constraint set (4), see Imai et al. ${ }^{(11)}$.

\subsection{Lagrangian relaxation}

As described in section 1, the heuristics proposed here are based on the subgradient procedure with Lagrangian relaxation of the original problem (DBAPD). The Lagrangian relaxation of DBAPD is as follows where $\lambda_{i j k}$ is a Lagrangian multiplier for berth $i$, ship $j$ and service order $k$.

[LR1]

Minimize $\sum_{i \in B} \sum_{j \in V} \sum_{k \in U}\left\{k C_{i j}+S_{i}-A_{j}\right\} x_{i j k}+\sum_{i \in B} \sum_{j \in V} \sum_{k \in U} k y_{i j k}$ $-\sum_{i \in B} \sum_{j \in W_{i}} \sum_{k \in U} \lambda_{i j k}\left\{\sum_{l \in V} \sum_{m \in P_{k}}\left(C_{i l} x_{i l m}+y_{i l m}\right)+y_{i j k}-\left(A_{j}-S_{i}\right) x_{i j k}\right\}$ subject to (2), (3), (5), (6)

In [LR1], $y_{i j k}$ is redundant since it is not included in (2), (3), (5) and (6). Therefore, eliminating $y_{i j k}$ from objective (7), it is transformed as follows:

[LR2]

$$
\begin{aligned}
\text { Minimize } & \sum_{i \in B} \sum_{j \in V} \sum_{k \in U}\left\{k C_{i j}+S_{i}-A_{j}\right\} x_{i j k} \\
& -\sum_{i \in B} \sum_{j \in W_{i}} \sum_{k \in U} \lambda_{i j k}\left(S_{i}-A_{j}\right) x_{i j k} \\
& -\sum_{i \in B} \sum_{j \in V} \sum_{k \in U} \sum_{l \in W_{i}} \sum_{m<k} \lambda_{i l m} C_{i j} x_{i j k}
\end{aligned}
$$

subject to (2), (3), (5), (6)

Furthermore, this turns to be as follows:

[LR3]

Minimize $\sum_{i \in B} \sum_{j \in V} \sum_{k \in U} E_{i j k} x_{i j k}$

subject to (2), (3), (5), (6)

where $E_{i j k}$ is a representative cost.

[LR3] can be easily reduced to the two dimensional assignment problem, which is easily solved by an efficient solution method such as the Hungarian method.

\section{Solution procedure}

\subsection{Subgradient optimization}

The quality of the feasible solution obtained using the above procedure is strongly dependent on one's ability to determine good Lagrangian multipliers, $\lambda_{i j k}$. Essentially, with a zero multiplier of $\lambda_{i j k}$ in [LR3] for each combination of $(i, j, k)$, we assume that ships can be allocated to berths in any order while minimizing the total service time including waiting and handling times. This may lead to an infeasible solution to [DBAPD] as some ships may be served before arrival. Since values of multipliers increase that correspond to the ships with infeasible berthing, i.e., the start of berthing ships served before arrival, is getting far from the arrival time, the cost, $E_{i j k}$, of those ships is increased in [LR3], probably resulting in later service of them. In other words, the solution to [LR3] gets closer to a feasible solution to [DBAPD].

Good multipliers are also important as the quality of the lower bound, i.e., the objective value of [DBAPD], is a function of these multipliers. We employ the subgradient optimization procedure to determine a good set of Lagrangian multipliers that results in tight lower and upper bounds. As this procedure is widely utilized for solving difficult combinatorial problems, only its outline is described below:

\section{[SUBGR]}

Step 1: Initialize multipliers $\lambda_{i j k}$.

Step 2: Construct a Lagrangian relaxation problem [LR3] with $\lambda_{i j k}$ and solve it optimally to obtain the lower bound to [DBAPD]. 
Step 3: Find a feasible solution to [DBAPD] from the optimal solution to [LR3] by a heuristic. The objective value of this solution defines the upper bound to [DBAPD]. If this upper bound is better than the best one obtained up to this iteration, update the best upper bound.

Step 4: Stop the procedure if a stopping criterion is met (e.g., the iteration limit is reached or the optimal solution to [DBAPD] is found).

Step 5: Update $\lambda_{i j k}$ by computing the step size and subgradient with the best upper bound identified so far and the lower bound obtained in this iteration. Go to Step 2.

For finding a feasible solution in Step 3 of [SUBGR], Imai et al. ${ }^{(1)}$ proposed three different heuristics: namely SIMPLE, INDIVIDUAL and INTERACT. The procedures of these heuristics with an optimal solution to [LR3] are summarized as follows:

SIMPLE: Services of those ships that are served before arrival in each berth in the solution to [LR3] are postponed up to arrival times. All ships after them in the solution are, in connection with them, also moved afterward without changing the service order.

INDIVIDUAL: This procedure performs the same as SIMPLE first. Then, if there is an idle time in each berth between consecutive two ships, appropriate ships after them are moved into the idle time as long as the consecutive ships and shifted ones are all feasible in terms of arrival time.

INTERACT: This procedure works the same as INDIVIDUAL first. Then, examining all possible swaps between two ships across different berths, perform swaps if the swaps result in a shorter overall service time.

As mentioned before, Imai et al. ${ }^{(11)}$ developed three subgradient procedures, each using different one of the three heuristics. The INTERACT procedure in that study is embedded in the INDIVIDUAL procedure. So, in INTERACT, ships allocated to each berth by SIMPLE are shuffled by INDIVIDUAL and also re-shuffled by adding some ships to the currently processed berth from other berths. That study found INTERACT not as good as INDIVIDUAL. In this study, all berths are shuffled by INTDIVIDUAL first. Then, the interchange of ships between berths is performed as INTERACT process.

\subsection{Improvement of heuristic}

In order to find a better feasible solution, this study will make a modification to Step 3 of [SUBGR], which was developed in Imai et al. ${ }^{(11)}$.

Taking a BAP only with a single berth as an example, we explain a concept of the modification. If the start of berth planning $S_{i}$ is larger than $A_{1}$, i.e., the planning gets started after all calling ships have arrived, the DBAPD becomes the static BAP in discrete locations (SBAPD). For the detail of SBAPD, see Imai et al. ${ }^{(11)}$. The SBAPD corresponds to a single machine scheduling problem with the objective of minimizing the total weighted completion time, i.e., 1||$\sum w_{j} F_{j}$, where all weight $w_{i}=1$ and $F_{j}$ is completion time of job $j$.

The problem of total weighted completion time finds an optimal solution by using the weighted shortest processing time first (WSPT) rule. The WSPT serves jobs in ascending order of their process times (handling time $C_{i j}$ in SBAPD).

Letting $C_{j}$ be a ship handling time in the single berth, the optimal solution to SBAPD can be found by serving ships in any order in case of a unit $C_{j}$ for all the ships. On the other hand, for different $C_{j}$ the optimal solution results from serving ships in ascending order of $C_{j}$.

For the DBAPD with a single berth, the optimal solution is found by FCFS (First-Come-First-Served) in case of unit handling time. A modification of the solution to the relaxed problem of DBAPD, i.e., SBAPD, leads to a feasible solution to DBAPD. Thus, a well organized solution to SBAPD in terms of ship arrival and berthing time results in a better feasible solution to DBAPD. However, as shown above, ships are served in arbitrary order in SBAPD of a unit $C_{j}$ case. Consequently, if we modify the solution to SBAPD to fit DBAPD by the SIMPLE heuristic, it does not likely result in a better DBAPD solution.

If we apply $w_{i}=1 / j$ for a single machine problem as the SBAPD, the weighted handling time $C_{j} / j$ is increasing with a decreasing ship number $j$. Note that ships are numbered in decreasing order of ship arrival, i.e., $A_{j} \leq A_{j^{\prime}}$ for $j>j^{\prime}$. Thus, a solution to the relaxed problem of DBAPD serves ships in ascending order of the weighted handling time, $C_{j} / j$, i.e., in 
FCFS rule. This computational property with $C_{i j} / j$ likely serves ships by FCFS for the multiple berth problem, making the approximate solution of DBAPD with $C_{i j} / j$ better than the one with $C_{i j}$. In favor of the above property, Step 3 in the subgradient procedure is modified as follows:

Step 3-1: Form DBAPD with $C_{i j} / j$ instead of $C_{i j}$ as follows:

$$
\begin{aligned}
\text { Minimize } & \sum_{i \in B} \sum_{j \in V} \sum_{k \in U}\left(k C_{i j} / j+S_{i}-A_{j}\right) x_{i j k} \\
+ & \sum_{i \in B} \sum_{j \in V} \sum_{k \in U} y_{i j k}
\end{aligned}
$$

subject to (2), (3), (5), (6)

Step 3-2: Given updated Lagrangian multipliers $\lambda_{i j k}$, form a Lagrangian relaxation [LR3] of the modified DBAPD in Step 3-1 and solve it optimally.

Step 3-3: From the optimal solution to [LR3], find a feasible solution to [DBAPD] with $C_{i j} / j$ by a heuristic. The objective value of this solution defines the upper bound to [DBAPD]. If this upper bound is better than the best one obtained up to this iteration, update the best upper bound.

As mentioned before, each of SIMPLE, INDIVIDUAL and INTERACT is applied to find a feasible solution in Step 3-3. Note that in the modified subgradient procedure above, the optimal solution to [LR3] in Step 3-2 does not provide the lower bound, which is defined from the objective value of solution to [LR3] in Step 2 as the original subgradient procedure. The reason is that [LR3] in Step 3-2 is not the relaxed problem to the DBAPD with $C_{i j}$.

\section{Numerical experiments}

\subsection{Settings}

We carry out numerical experiments with the following settings. All computational instances assume four berths with 100 calling ships. 18 different calling ship scenarios are prepared with various calling interval and cargo handling times. For each computation instance of the 18 ones, we set three different start times of planning horizon, $S_{i}$, where all berths have the same value of $S_{i}$. We prepare three different values for $S_{i}$. The earliest time is equivalent to the first quarter of the 100 ships (S1), the next is the half (S2) and the third is the three quarters (S3). The combination of three different $S_{i}$ times 18 scenarios leads to 54 problem instances in total.
The ship calling interval time is randomly generated based on an exponential function with the average interval times of 1,5 and $8 \mathrm{~h}$. The cargo handling time of a ship varies on its potential berthing locations, but it is generated based on uniform random variables with different average times of 4 and $12 \mathrm{~h}$. Also, we prepare three different fluctuations between the maximum and minimum amounts of the cargo handling time of a ship: 0,100 and $200 \%$ of the average time. We used three different weighted handling times: $C_{i j} / j, C_{i j} /\left(j^{*} j\right)$ and $C_{i j} / \log (j)$.

\subsection{Results}

First, we look at the average value of all computation instances. TABLE 1 and Fig. 2 show the average GAP ( $\left.=(\mathrm{UB}-\mathrm{LB})^{*} 100 / \mathrm{LB}\right)$ where $\mathrm{UB}$ is the upper bound and LB is the lower bound. The GAP guarantees the worst case quality of the solution against the optimal solution. Generally, the quality of approximate solutions should be measured by the GAP between the approximate and optimal solutions. However, for practically large problem instances the optimal solution is difficult to be found. As the LB is the lowest bound of the optimal solution value, it is common to use the LB for substitution of the optimal value. Notice that in the worst case of the computation instances we obtained a negative value of LB. Those instances are excluded from the GAP analysis.

Looking at TABLE 1, it is found that SIMPLE is worst and INTERACT is best among the three heuristics. INDIVIDUAL is in the middle between the others, but the difference between INDIVIDUAL and INTERACT is quite small.

With the original $C_{i j}$, there is a large difference in GAP between SIMPLE and the others. However, any weighted handling times improve the GAP of SIMPLE to a great extent. Comparing different weighted handling times, $C_{i j} /\left(j^{*} j\right)$ is best for SIMPLE, whilst $C_{i j} / \log (j)$ is best for both INDIVIDUAL and INTERACT.

TABLE 2 illustrates UB. The trend for UB is the same as for GAP. If we compare the different heuristics for UB with a specific weighted handling

\begin{tabular}{lcccc} 
& \multicolumn{5}{c}{ TABLE 1. GAP(\%) } \\
& $\mathrm{C}$ & $\mathrm{C} / \log \mathrm{j}$ & $\mathrm{C} / \mathrm{j}$ & $\mathrm{C} / \mathrm{j} * \mathrm{j}$ \\
\hline SIMPLE & 622.2 & 373.5 & 235.0 & 204.0 \\
\hline INDIVIDUAL & 188.7 & 177.8 & 178.3 & 180.4 \\
\hline INTERACT & 178.7 & 176.7 & 176.7 & 179.9 \\
\hline
\end{tabular}


TABLE 2. UB (h)

\begin{tabular}{lcccc} 
& $\mathrm{C}$ & $\mathrm{C} / \operatorname{logj}$ & $\mathrm{C} / \mathrm{j}$ & $\mathrm{C} / \mathrm{j} * \mathrm{j}$ \\
\hline SIMPLE & 13929.3 & 9555.8 & 7847.9 & 7354.6 \\
\hline INDIVIDUAL & 7125.4 & 6864.5 & 6905.5 & 6946.0 \\
\hline INTERACT & 6877.8 & 6856.0 & 6871.1 & 6946.8 \\
\hline
\end{tabular}

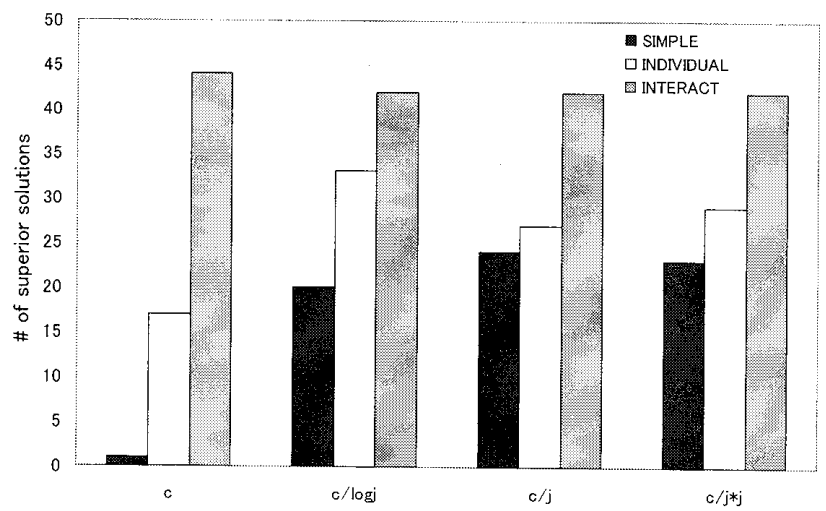

Fig. 2 Number of superior solutions among different weighted handling times as for UB

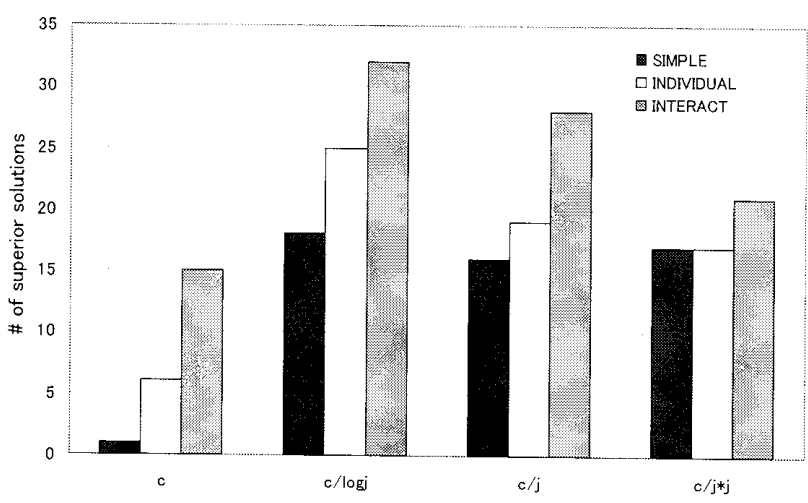

Fig. 3 Number of superior solutions among all different heuristics and weighted handling times as for UB

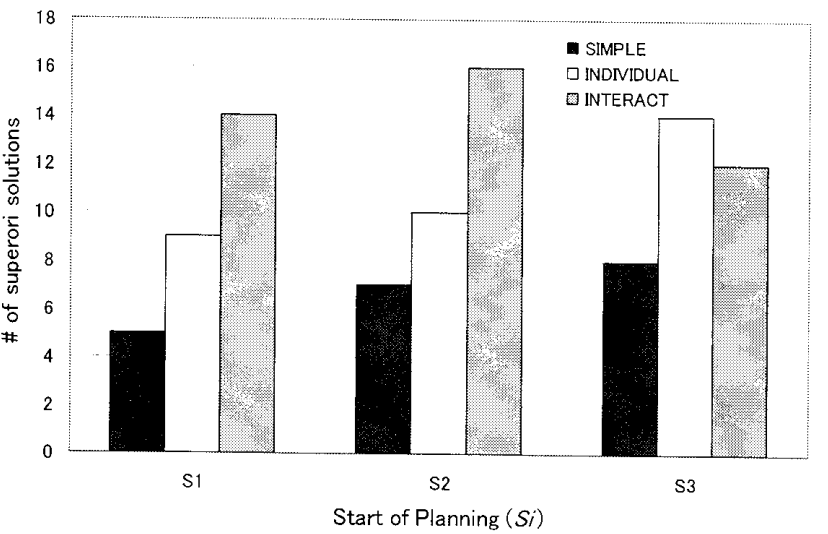

Fig. 4 Number of superior solutions among all different heuristics with $C_{i j} / \log (j)$ at different $S_{i}$ as for UB

time by using TABLE 2, INDIVIDUAL is best for $C_{i j} /\left(j^{*} j\right)$, while INTERACT is best for the others.

From the above discussion with GAP and UB, the algorithms with modified weighted handling times produce better solutions than the existing [SUBGR], while the solution improvement with heuristics INDIVIDUAL and INTERACT is not so significant.

As observed previously, the difference in UB (and GAP) between INDIVIDUAL and INTERACT is quite minor, as UB examined is an average value over the 54 problem instances. Therefore, next we examine the performance of different heuristics with different weighted handling times from the viewpoint of each individual problem instance. For this, we look into the superiority of heuristics by the number of superior solutions for UB. We score the number of the best solutions among different heuristics with each of the four different weighted handling times for each problem instance. The total scores of the three heuristics over the 54 problem instances by four different weighted handling times are demonstrated in Fig. 2. For each weighted handling time, INTERACT has the largest score, which is nearly double of the one of INDIVIDUAL.

Fig. 3 shows the score as the number of superior solutions for all combinations of three heuristics and four weighted handling times. In this comparison, INTERACT is best among 12 different combinations. In this result, modified algorithms are better than the existing ones. The best weighted handling time for INTERACT is $C_{i j} / \log (j)$.

Fig.4 illustrates the number of superior solutions by the three heuristics with $C_{i j} / \log (j)$ for different start times of planning, $S_{i}$. It is found that with earlier start times INTERACT outperforms, while with a later start time INDIVIDUAL is better. This is also the trend for heuristics with other weighted handling times.

Notice that the CPU times of the three heuristics with a specific weighted handling time are almost the same. From the above discussions, we can conclude that INTERACT with $C_{i j} / \log (j)$ is the best heuristic among others.

\section{Conclusions}

In this study, we discussed about a modification of subgradient procedure with Lagrangian relaxation for the DBAPD by introducing a weighted handling time. In the modified procedure, a relaxed problem with this weighted handling time was solved and its solution 
was converted to a feasible solution of the DBAPD. Note that this relaxed solution was never used for Lagrangian multiplier update. The reason is that strictly speaking this relaxed problem is a pseudo relaxation and not a true relaxation to the DBAPD because of the weighted (not original) handling time.

We carried out a wide variety of numerical experiments, which showed that the modification produced better solutions than the existing algorithms. Among 12 different heuristics with various weighted handling times, heuristic INTERACT with $C_{i j} / \log (j)$ was best although INDIVIDUAL was better only with a late start time of the planning.

This research is supported by the JSPS Grant-inAid for Scientific Research-C Grant 22510149.

\section{References}

(1) J.F. Alvarez, T. Longva and E.S. Engebrethsen: A Methodology to Assess Vessel Berthing and Speed Optimization Policies, Maritime Economics and Logistics, Vol.12, No.4, pp.327-346, 2010.

(2) K. Buhrkal, S. Zuglian, S. Ropke, J. Larsen and R. Lusby, Models for the Discrete Berth Allocation Problem: a Computational Comparison, Transportation Research Part-E, Vol.47, pp.461-473, 2011.

(3) D. Chang, W. Yan, C.-H. Chen and Z.A. Jiang: Berth Allocation Strategy Using Heuristics Algorithm and Simulation Optimisation, International Journal of Computer Applications in Technology, Vol. 32, No.4, pp.272-281, 2008.

(4) J.-F. Cordeau, G. Laporte, P. Legato and L. Moccia: Models and Tabu Search Heuristics for the Berth-allocation Problem. Transportation Science, 39, pp.526-538, 2005.

(5) G. Giallomobardo, L. Moccia, M. Salani and I. Vacca: Modeling and Solving the Tactical Berth Allocation Problem, Transportation Research Part-B, Vol.44, pp.232-245, 2010.

(6) M.M. Golias, M. Boile and S. Theofanis: Berth Scheduling by Customer Service Differentiation: A Multi-objective Approach, Transportation Research Part-E, Vol.45, pp.878-892, 2009.

(7) M.M. Golias, M. Boile and S. Theofanis: A Lamda-optimal Based Heuristic for the Berth Scheduling Problem, Transportation Research
Part-C, Vol.18, pp.794-806, 2010.

(8) Y. Guan, W.-Q. Xiao, R.K. Chueng and C.-L. Li: A Multiprocessor Task Scheduling Model for Berth Allocation: Heuristic and Worst Case Analysis. Operations Research Letter, Vol.30, pp.343-350, 2002.

(9) P. Hansen, C. Oguz and N. Mladenovic: Variable Neighborhood Search for Minimum Cost Berth Allocation, European Journal of Operational Research, Vol.191, pp.636-649, 2008.

(10) A. Imai, K. Nagaiwa and W.T. Chan: Efficient Panning of Berth Allocation for Container Terminals in Asia, Journal of Advanced Transportation, Vol.31, pp.75-94, 1997.

(11) A. Imai, E. Nishimura and S. Papadimitriou: The Dynamic Berth Allocation Problem for a Container Port, Transportation Research Part-B, Vol.35, pp.401-417, 2001.

(12) A. Imai, E. Nishimura and S. Papadimitriou: Berth Allocation with Service Priority, Transportation Research Part-B, Vol.37, pp.437-457, 2003.

(13) A. Imai, E. Nishimura and S. Papadimitriou: Corrigendum to "The dynamic berth allocation problem for a container port" [Transportation Research Part B 35 (2001) 401-417], Transportation Research Part-B, Vol.39, p.197, 2005.

(14) A. Imai, E. Nishimura and S. Papadimitriou: Berth Allocation in a Container Port: Using a Continuous Location Space Approach, Transportation Research Part-B, Vol.39, pp.199-221, 2005.

(15) A. Imai, E. Nishimura, M. Hattori and S. Papadimitriou: Berth Allocation at Indented Berths for Mega-containerships, European Journal of Operational Research, Vol.179, pp.579-593, 2007.

(16) A. Imai, E. Nishimura and S. Papadimitriou: Berthing Ships at a Multi-user Container Terminal with a Limited Quay Capacity, Transportation Research Part-E, Vol.44, pp.136-151, 2008.

(17) K.H. Kim, and K.C. Moon: Berth Scheduling by Simulated Annealing, Transportation Research Part-B, Vol.37, pp.541-560, 2003.

(18) Y. Lee and C.-Y. Chen: An Optimization 
Heuristic for the Berth Scheduling Problem, European Journal of Operational Research, Vol.196, pp.500-508, 2009.

(19) D.-H. Lee, J.H. Chen and J.X. Cao: The Continuous Berth Allocation Problem: A Greedy Randomized Adaptive Search Solution, Transportation Research Part-E, Vol.46, pp.1017-1029, 2010.

(20) C.-L. Li, X. Cai and C.-Y. Lee: Scheduling with Multiple-job-on-one-processor Pattern, IIE Transaction, Vol.30, pp.433-445, 1998.

(21) A. Lim: The Berth Planning Problem, Operations, Research Letters, Vol.22, pp.105-110, 1998.

(22) M.F. Monaco and M. Sammarra: The Berth Allocation Problem: A Strong Formulation Solved by a Lagrangean Approach, Vol.41, No.2, pp.265-280, 2007.

(23) R. Moorthy and C.-P. Teo: Berth Management in Container Terminal: The Berth Template Design Problem, OR Spectrum, Vol.28, pp. 495-518, 2006.

(24) E. Nishimura, A. Imai and S. Papadimitriou: Berth Allocation Planning in the Public Berth System by Genetic Algorithms, European Journal of Operational Research, Vol.131, pp.282-292, 2001.

(25) K.T. Park and K.H. Kim: Berth Scheduling for Container Terminals by Using a Sub-gradient Optimization Technique, Journal of the Operational Research Society, Vol.53, pp.1054-1062, 2002.

(26) G.K.D. Saharidis, M.M. Golias, M. Boile, S. Theofanis and M.G. Ierapetritou: The Berth Scheduling Problem with Customer Differentiation: A New Methodological Approach Based on Hierarchical Optimization, International Journal of Advanced Manufacturing Technology, Vol.46, pp.377-393, 2010.

(27) F. Wang and A. Lim: A Stochastic Beam Search for the Berth Allocation Problem, Decision Support Systems, Vol.41, pp.2186-2196, 2007.

(28) D. Xu, C.-L. Li and J. Y.-T. Leung: Berth Allocation with Time-dependent Physical Limitations on Vessels, European Journal of Operational Research, Vol.216, pp.47-56, 2012.

\section{Questions \& Answers}

Toshihiko MATSUO (Tokai University):

Q1. What is the difference between the existing method and modified method used in this study?

Q2. It is expected that CPU time exponentially increases with the increase of the problem size, e.g. with the number of calling ships. How was the CPU time from this point of view?

Yukiko YAMAKAWA (Kobe University):

A1. The DBAPD serves ships in ascending order of handling time and arrival time. However, the existing method mainly focuses on only the handling time. In order to obtain a better solution, we modified the existing method by taking into account the factor of First-Come-First-Served (FCFS). For this modification, we made a relaxed problem by using the modified handling time in its objective function, i.e., the ships' handling time divided by ship numbers that are given in descending order of their arrival times. Then, the optimal solution to this relaxed problem more likely satisfies the ship handling time and FCFS factors than the original relaxed problem. In fact, we used other functions of ship numbers for the modified handling time in the numerical experiments such as $C_{i j} / \log (j)$ and $C_{i j} /\left(j^{*} j\right)$.

A2. For the numerical experiments, we used problem instances with only 100 ships, so we did not calculate other ship number cases. With 100 ships, the CPU time is less than 60 seconds for the existing method and about 200 seconds for the modified methods. Note that the existing method produces worse solutions with shorter CPU time than the modified methods. Solutions with $C_{i j} / \log (j)$ is best. The time is not so different among three heuristics, SIMPLE, INDIVIDUAL and INTERACT. 